hospitals realized which cases they should transfer immediately. In most cases it takes no more than two hours to move a patient from any hospital to a major centre, and it is in this area of improved transport of a patient to the major centres that the greatest improvement in our treatment could be achieved. A railway ambulance car would help more than any other single thing in an island such as ours.

One of the problems that always besets teachers is to know what the student should be taught. I think that the basis of what we should teach every student, certainly as far as trauma is concerned, is what every doctor should know. Whether he be a general surgeon or physician or gynaecologist or orthopaedic surgeon, there are certain basic principles in treatment of patients which all of us should know and that is what should be implanted in our students quite firmly. We have to resist the tendency to try and make them into miniature specialists or replicas of ourselves.

The next important area where knowledge should lead to wisdom is that of continuing education. The student must be made to realize from the very beginning that all his teachers are trying to do is to set him on a course which he will continue for the rest of his life-namely, self-education. This implies the attendance at meetings and regular medical conferences. It is unfortunate that those who attend conferences really need to attend least. It is those who do not like going, who seldom read their journals, and are rapidly out of date, and then are afraid to go in to meet their peers because it is obvious that their knowlege is poor, who debase the standard of patient care.

The thought that I would like to leave with you is contained in a verse from Couper:

"Knowledge and wisdom, far from being one,

Have 'oft times no connexion. Knowledge dwells

In heads replete with thoughts of other men,

Wisdom in minds attentive to their own.

Knowledge is proud that he has learned so much,

Wisdom is humble that he knows no more."

If we humbly look into our patients' needs and realize that that is what this conference, all conferences, and teaching is about, we will indeed have learnt a great deal.

\title{
Abdominal Injuries
}

\section{A. D. ROY}

British Medical fournal, 1974, 4, 335-336

The abdomen is easily injured, and, when threatened, one's instinct is to protect it because it contains vital organs. It can be penetrated by weapons or missiles, or damaged by blunt trauma. In this violent world the incidence of abdominal injuries is rising and there are three main causes: road traffic accidents; industrial and domestic accidents; and civil and military violence.

The first cause is by far the most common in most parts of the world, and may be regarded as a disease which has reached pandemic proportions. Local epidemics of civil and military violence alter the pattern, but these are usually temporary episodes. Until 1915 the recognized treatment of both closed and open injuries of the abdomen was conservative, operation was not advised, and the mortality rate was very high. In fact, the surgery of the injured abdomen has a very short history.

The division of injuries into those that are due to blunt injury, and are therefore closed, and those that are due to the penetration of the abdomen by a sharp object or missile, is valid. Injury to the adjacent parts of the body, or even occasionally remote areas, may involve trauma to the abdomen: a missile may enter the upper thorax and yet penetrate the abdominal cavity. Conversely, injury which is apparently at first confined to the abdomen may involve injury to other areas-for instance, rupture of the diaphragm is not uncommonly the result of blunt injury to the body. When the injury is closed the trunk and pelvis should be carefully examined for bruising and abrasions to determine how the trauma had been applied. Fractures of the lower ribs will indicate the likelihood of injury to liver and spleen, while fractures of the pelvic girdle will indicate the possibility of injury to the bladder or urethra. Fractures of the transverse spinal processes are often associated with injuries to the kidney. In missile injuries the entry and exit wounds should

Queen's University of Belfast, Belfast

A. D. ROY, M.B., F.R.c.s., Professor of Surgery be correlated with the probable passage through the body taking into account any bones which may have deflected the missile. If there is no exit wound the position of the missile in the body must be determined.

Head injuries may conceal a major abdominal injury. Loss of consciousness and flaccidity of muscles will conceal the symptoms and signs and the need for craniotomy or respiratory support may distract attention away from the abdomen. Patients with head injuries should always have a careful examination of the abdomen and it must be emphasized again that head injuries do not cause hypotension. If a patient with a head injury is shocked it is probably because of some other lesion and this possibility should be carefully investigated.

\section{Resuscitation}

Resuscitation must be started immediately the patient is admitted, and go on while the injury is being assessed. With one exception the patient should be stabilized before laparotomy is carried out. This exception is when a major vessel has been damaged: in this case the haemorrhage must be controlled rapidly. A quick laparotomy to stop haemorrhage can be followed by a pause for resuscitation before repair of the injury proceeds.

Closed injuries demand an early decision for or against laparotomy and, in general, if any doubt remains whether there is damage to a solid or hollow viscus or whether haemorrhage has occurred then laparotomy should be performed. Needle aspiration of the four quadrants of the abdomen is safe and a positive result is valuable. The aspiration of either blood, gas, or faecal matter is significant. A negative result does not exclude the need for laparotomy. Plain radiography of the abdomen is less helpful. Evidence of free gas, or in late cases of ileus, may be helpful, but a negative reading of the $x$-ray films should not be allowed to override clinical signs.

Open or penetrating injuries due to missiles, knives, or other sharp objects must be explored. Missile injuries are the most complex because of the deflection of the missile in the tissues and the velocity of the missile. Low velocity 
missiles form a track through the tissues with a small area of bruising and damage around it. High velocity missiles, on the other hand, as they slow down in the tissues, disperse large quantities of kinetic energy into the surrounding tissues and cause widespread disruption and necrosis-extending several centimetres away from the track itself. This may necessitate wide resection of tissues which will necrose if left in the body-for example, a high velocity bullet passing through a lobe of the liver may disrupt as much as twothirds of the liver substance and necessitate excision of at least one lobe of the liver. The more solid the tissue the greater the area of damage will be.

Penetrating injuries caused by knives or other sharp objects are more predictable. Quite often they do not penetrate the abdominal wall and conservative treatment has been advocated for some of them. It is never possible to be certain that the peritoneal cavity has not been penetrated and all these wounds should be explored under anaesthesia and the abdomen opened if there is any doubt at all about the depth of penetration. A penetrating injury may occur without an external wound. Missiles and sharp objects may enter the abdominal cavity through the rectum and vagina, and compressed air may also cause a rupture of the rectum when applied to the anus by accident (or a ghoulish joke).

\section{Treatment}

If a decision is made not to explore the abdomen this must be reviewed every six hours for at least 48 hours and the decision reversed if there is a rise in pulse rate, an increase in abdominal pain, or other sign of a progressive intraabdominal lesion.

The most urgent laparotomies are for haemorrhage. Haemorrhage may be due to injury to liver, spleen, or kidney, or to the division (partial or complete) of a large vein or artery. If, clearly, attempts at resuscitation are not succeeding, because the loss of blood is outstripping measures to maintain blood volume, the patient must be rapidly prepared for laparatomy. The abdomen should be opened through an incision as close as possible to the probable site of bleeding and the haemorrhage controlled by finger pressure, packing, or clamping - whichever is the most appropriate. At this point resuscitation can be completed and the operation be resumed only when the patient's condition is stable.

Repair of major vessels may be difficult. Access must be as complete as possible and repair may require the use of prosthesis or synthetic patches. The hepatic veins and the inferior vena cava in its course behind the liver are the most difficult to deal with and will necessitate a thoracoabdominal incision.

\section{DAMAGE TO VISCERA}

Repair of damage to the liver can be achieved by careful insertion of mattress sutures tied over a patch of free muscle. Nevertheless, if the laceration is deep and a large area of liver is devitalized then partial hepatectomy is essential-usually requiring a thoracoabdominal incision. There is no case for packing a lacerated liver; it may control bleeding at the time but is usually followed by infection and secondary haemorrhage.

Any damage to spleen should be treated by splenectomy. This is usually easy but a lacerated, enlarged, abnormal spleen may be difficult to remove if there are many adhesions between it and the diaphragm. In such cases the splenic artery should be ligated at the upper border of the pancreas before the spleen is mobilized.

A damaged kidney should be repaired if at all possible. Only if there is a hilar tear affecting the vessels is nephrectomy indicated, and in all cases of suspected renal damage, where laparotomy is required, it is essential that an emergency intravenous pyelogram should be performed to confirm that the undamaged kidney is functioning normally. A damaged kidney requires exploration only if haemorrhage is severe or if there is evidence of extravazation of urine.

Injuries to bowel or bladder must all be found and repaired. In all cases the most careful examination of the alimentary canal from stomach to rectum must be carried out. Certain parts of the intestine are difficult to examine and this particularly applies to the distal half of the duodenum. In closed injuries the junction between fixed and mobile parts of the intestine are particularly likely to be torn. Resection may, therefore, be better than repair if there is any doubt about viability. Most cases are seen very soon after injury and primary closure of large bowel injuries, provided they are not too lacerated, has been the policy. Nevertheless, a recent review of the results has shown that, though mortality has not been high, the morbidity of primary closure has been considerable. My colleagues have, therefore, returned to the opinion that exteriorization of large bowel or defunctioning of the repaired intestine is a better policy. Injuries to the bladder are less common and repair should always be followed by a period of bladder drainage by catheter or suprapubic cystostomy.

Access to intra-abdominal injuries must be good. The pattern of injury will seldom conform to a standard incision; so the initial incision should be placed as close as possible to where the damage is likely to be. After initial exploration the incision will often require to be extended, of ten into the thoracic cavity, and one should not hesitate to do so if it is required for adequate exposure. Injury to the kidneys is better approached from the front as it is easier to gain quick access to the vessels. Injury to the liver and, especially, to the hepatic veins and the inferior vena cava will always require a thoracoabdominal approach.

\section{Postoperative Management}

After the initial laparotomy and repair great care should be taken with the postoperative management. Any possibility of a further, overlooked lesion will require a second exploration. Concern with the abdominal condition should not be allowed to distract attention from the respiratory and cardiovascular systems. The prophylactic use of antibiotics is possibly of little value. When sepsis complicates recovery then it will usually be Gram-negative organisms that are involved and the antibiotics should be carefully chosen to be effective against them. The Gram-negative, anaerobic organisms, Bacteriodes, are the most dangerous and should be treated with tetracycline or lincomycin. Bacteraemic shock usually results from a leaking anastomosis and will require support for respiration and circulation in an intensive care unit and, often, re-exploration of the abdomen.

The prophylactic use of antibiotics is probably of little value. It does not prevent wound infection and peritoneal sepsis is usually due to leakage from bowel in the postoperative period. Soiling of peritoneum at the time of wounding or operation is probably of little importance and I would treat soiling by washing the peritoneal cavity out with saline and not with a solution of antibiotics or other antibacterial agents. If sepsis does occur it should be treated appropriately. If there is any likelihood of leakage from the intestine the abdomen should be reopened for a further repair or resection of damaged intestine and drainage of collections of pus.

All injuries are preventable and it should be our aim to achieve this. Abdominal injuries, when they occur, are serious. Mortality and morbidity can be reduced by early care, careful diagnosis, scrupulous exploration, and repair of damaged organs and close supervision of the period of recovery. 